\title{
RADIOGRAPHIC ESTIMATION OF VERTEBRAL ROTATION IN SCOLIOSIS
}

\author{
M. H. Mehta, Calcutta, India
}

From the Institute of Orthopaedics, Royal National Orthopaedic Hospital, London and Stanmore

Radiographs taken under standard conditions play an important role in the management of scoliosis. Since the introduction by Ferguson (1949) and Cobb (1948) of their respective methods for measurement of the degree of lateral curvature in scoliosis, progressive deformity is assessed by changes in the curve angle, but increasing vertebral rotation is often overlooked for the lack of a suitable method to measure it. The need for rotational evaluation has long been recognised, but few attempts have been made to devise means to measure it.

Of the existing methods the two that are sometimes used are those of Cobb (1948) and of Nash and Moe (1969). In both, a single vertebral landmark is used as a guide to rotationthe spinous process in the former, the convex side pedicle in the latter. In neither method, however, is it possible to follow the chosen landmark through the full range of vertebral rotation of 0 to 90 degrees that can occur in scoliosis.

It was therefore decided to take radiographs of normal vertebrae at specified intervals of rotation and study the changing inter-relationship of the several parts of the vertebra to one another, with two objectives. Firstly, to determine whether the resulting radiographs are sufficiently distinctive in appearance to enable their easy identification one from the other; and secondly, to find out whether rotated apical vertebrae of scoliosis bear resemblance to normal vertebrae in rotation and, if they do, to assess the feasibility of using an image-matching radiographic technique to estimate rotation in scoliosis.

\section{MATERIAL AND METHOD}

Two short columns of vertebrae, the sixth to tenth thoracic and the first to fourth lumbar, from a young adult cadaver were separately mounted on a board by a screw passed through the lowest vertebra in such a way that the column could pivot around the screw. The pedicle and transverse process were selected as identifying landmarks.

Radiographs were taken with the $\mathrm{x}$-ray tube stationary, starting with an unrotated anteroposterior view. The vertebrae were then rotated clockwise-that is, in the direction of rotation which would be expected in a curve with its convexity to the right-and radiographs were taken at 15 degree intervals through an arc of 90 degrees. To facilitate identification the right or "convex side" pedicle of alternate vertebrae was marked by a radiopaque lead pellet placed on the upper edge of the pedicle at its junction with the vertebral body. A similar but smaller pellet was affixed to the tip of the right or convex side transverse process of the same vertebrae.

\section{RESULTS}

Radiographs of thoracic and lumbar vertebrae at 15-degree intervals of rotation resulted in a series of images, each with a distinctive appearance. Attempts to reduce the interval between successive radiographs to less than 15 degrees failed to yield images with the clearcut differences necessary for easy identification. The radiographs of thoracic vertebrae (T.6 to T.10) taken at 15-degree intervals of rotation are reproduced in Figures 1 to 7 . They illustrate the changing relationship of vertebral features to one another at different degrees of rotation. While several landmarks play their part in producing a composite picture particular attention is drawn, in the identification of each rotation view, to three: the pedicle, 


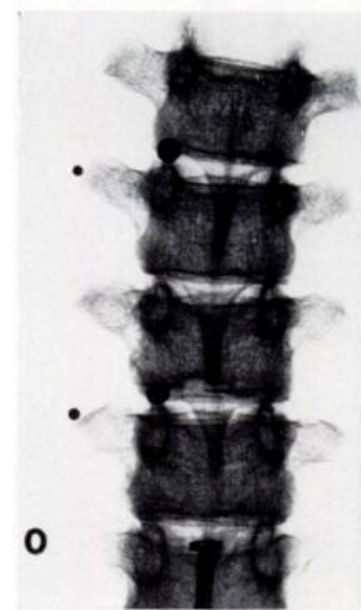

FIG. 1

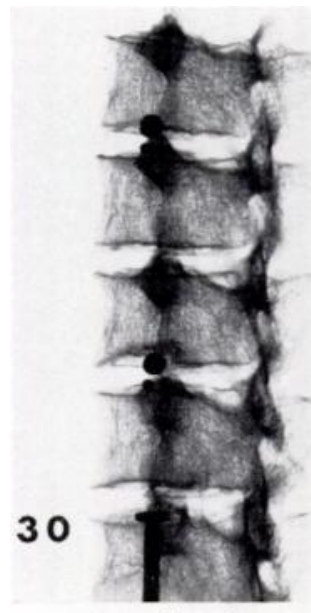

FIG. 3

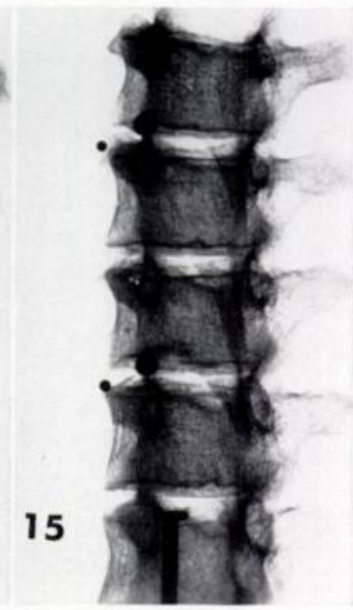

FIG. 2

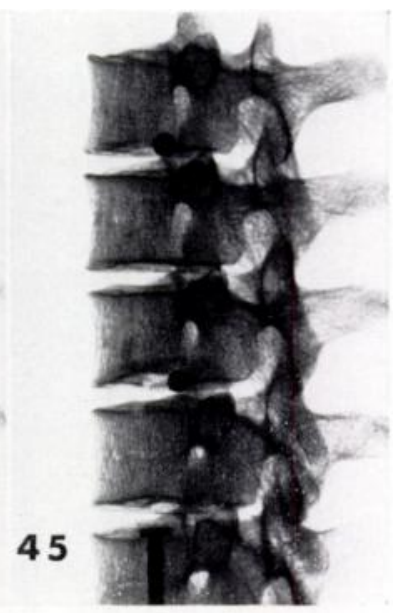

FIG. 4

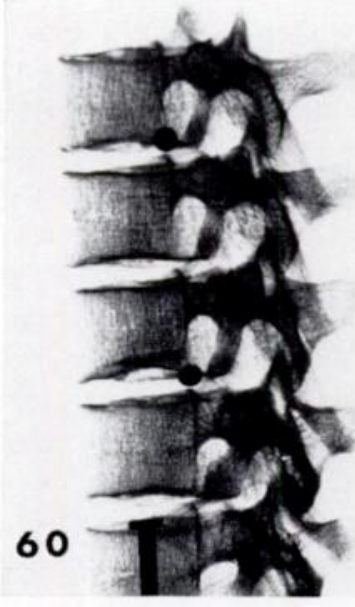

FIG. 5

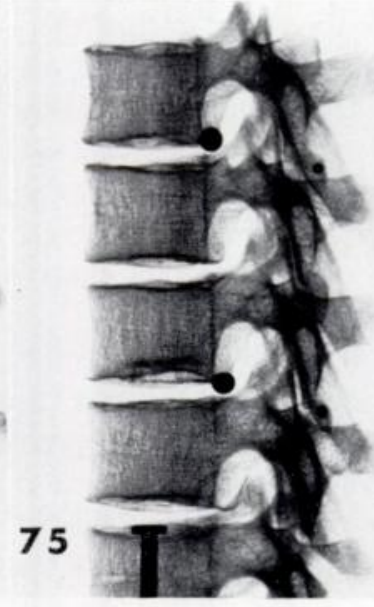

FIG. 6

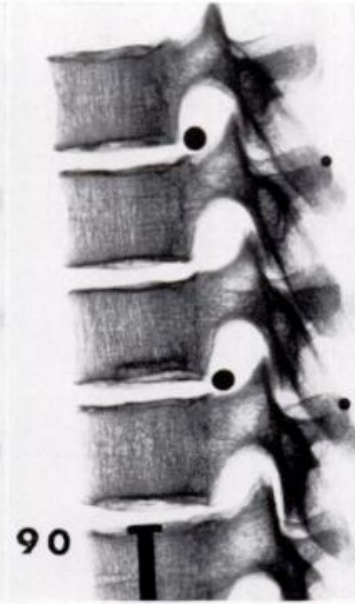

FIG. 7

Radiographs of thoracic vertebrae (T.6-10) taken at 15-degree intervals of rotation from 0 to 90 degrees. 


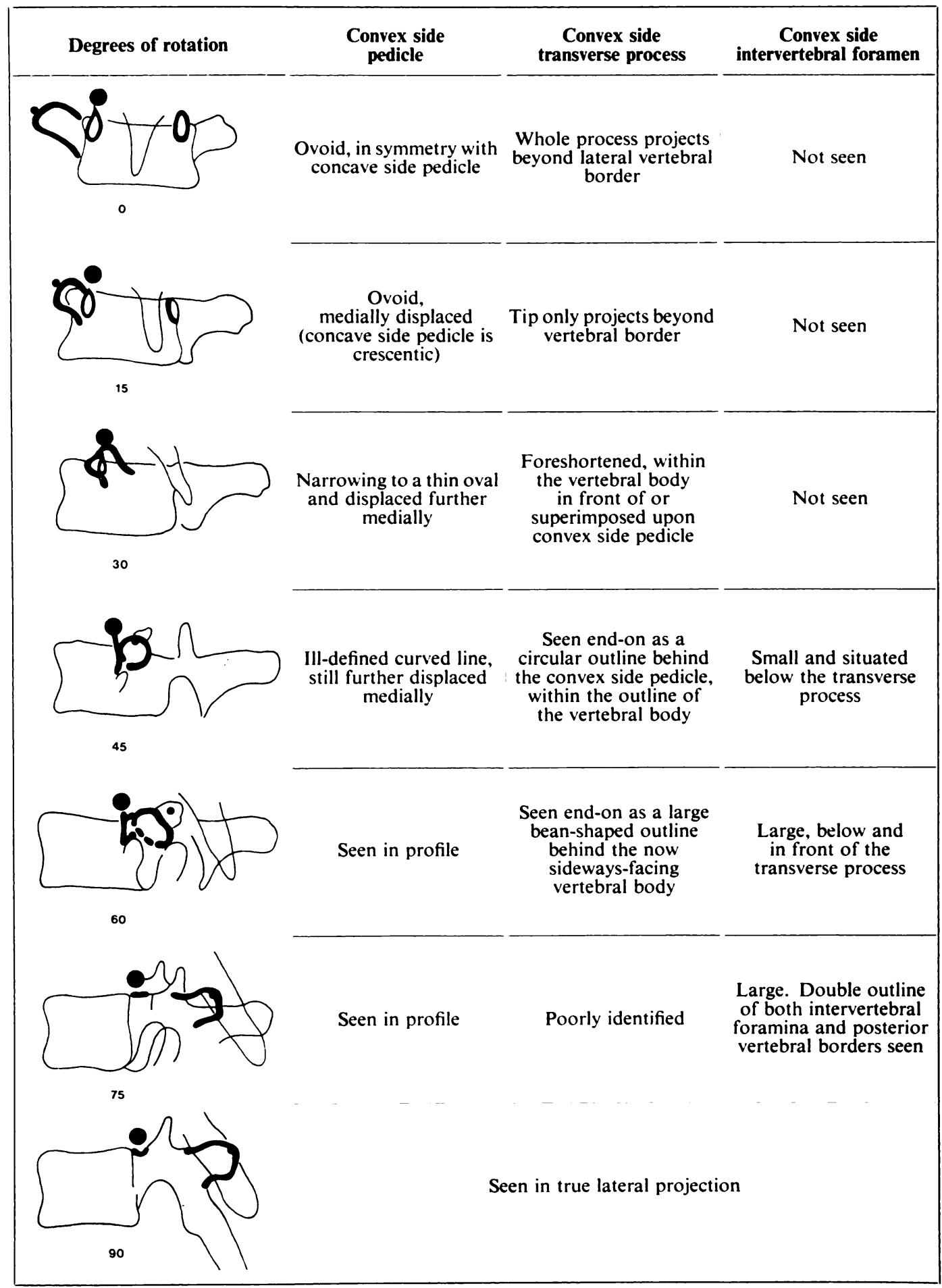

FIG. 8

Line drawings of radiographs shown in Figures 1 to 7 illustrating the main identifying features at different stages of rotation.

VOL. 55 B, No. 3, AUGUST 1973 
the transverse process and the intervertebral foramen of the convex side. Figure 8 shows these in line drawing and summarises the important aspects of each rotational view.

Figures 1 to 8 show that at 15 and 30 degrees the most important identifying landmark is the convex side pedicle, aided by the convex side transverse process. The progressive shortening of the latter helps to differentiate between 15- and 30-degree rotations. After 30 degrees the convex side pedicle is no longer of much help in identifying rotation; at 45 and 60 degrees it casts a poorly defined linear shadow and the dominant landmark is now the convex side transverse process seen "end-on". It is the relationship of the transverse process to the intervertebral foramen and to the outline of the vertebral body that enables one to differentiate between 45 - and 60 -degree rotations. At 45 degrees the large and roughly circular outline of the transverse process is situated above a small intervertebral foramen, both being contained within the outline of the vertebral body. At 60 degrees the now somewhat bean-shaped outline of the transverse process is above and behind an enlarged intervertebral foramen and both are placed well behind the outline of the vertebral body. The 75- and 90-degree views resemble each other: the differentiating feature between them is that at 75 degrees both convex and concave side margins of the posterior surface of the vertebral body are seen, whereas at 90 degrees the vertebral body is seen in true lateral projection.

From the foregoing it is apparent that there are similarities in appearance at 15 and 30 degrees, 45 and 60 degrees and 75 and 90 degrees, but there are also distinct differences between them which enable correct identification of each view.

For reasons of space the radiographs of the lumbar vertebrae in rotation have not been reproduced. Both lumbar and thoracic rotation series are essentially similar, but morphological differences in the relative size of transverse process and pedicle in thoracic and lumbar vertebrae give rise to minor differences in the radiographic appearance. In the thoracic vertebrae the pedicles are small and ovoid, while the transverse processes are substantial, club-shaped and are directed backwards and laterally from the vertebral arch at an angle of about 45 degrees. Consequently, the circular outline of the thoracic transverse process seen "end-on" at 45-degree rotation (Fig. 4) is larger than that of the ovoid pedicle seen at 0 -, 15- and 30-degree rotation (Figs. 1 to 3). In the lumbar vertebrae the situation is reversed: the pedicles are large and ovoid whereas the transverse processes are small and flattened from before backwards. Hence the pedicle is seen as a large ovoid shadow at 0,15 and 30 degrees, and the transverse process seen "end-on" at 45- and 60-degree rotation appears as a small oval shadow which is only half the size of the pedicular outline.

The further objective of this study was to discover whether rotated apical vertebrae in scoliosis project similar images and to determine to what extent, if any, the tilt and torsional changes within the body and neural arch elements of a scoliotic vertebra alter its rotational radiographic appearance from that of the normal. Comparison of radiographs of segments of a disembodied scoliotic spine with those of the normal spine in like degrees of rotation would have been the most direct way to determine this, but the difficulty in obtaining such a spine for study can be appreciated. It was therefore decided to employ an indirect method of comparison by examining the antero-posterior radiographs of children of all ages and with different degrees of idiopathic and paralytic scoliosis for the features of vertebral rotation. Radiographs of congenital scoliosis and severe kyphoscoliosis were not included.

Familiarity with the characteristic features of each phase of rotation in the normal spine enabled one to detect the same characteristics in the apical vertebrae regardless of the severity of the angle of curvature or the degree of rotation present. The close similarity between normal and apical vertebrae in the early stages of rotation in mild to moderate curves was not altogether unexpected; what was surprising was to find that, despite the structural changes which must exist in the vertebrae of severe and long-standing curves, the features characteristic of vertebral rotation at 45 degrees and over were present without significant alteration from the normal. Furthermore, these features were seen not only in the radiographs of older 
children nearing skeletal maturity (Figs. 9 to 13) but also in those of infants and young children in whom ossification of the cartilaginous neuro-central junction had not yet occurred (Figs.

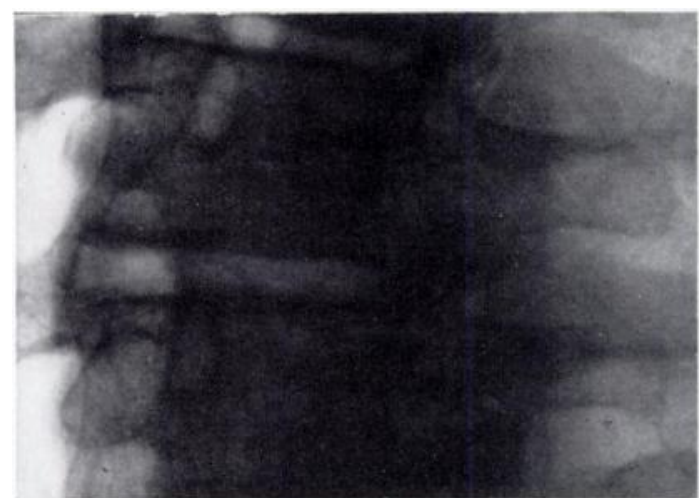

FiG. 9

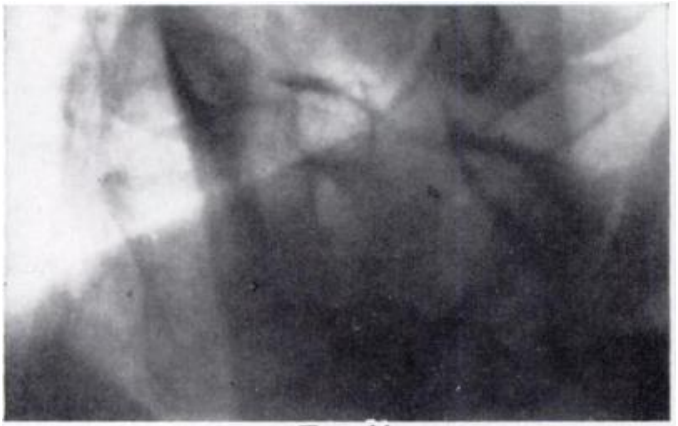

Fig. 11

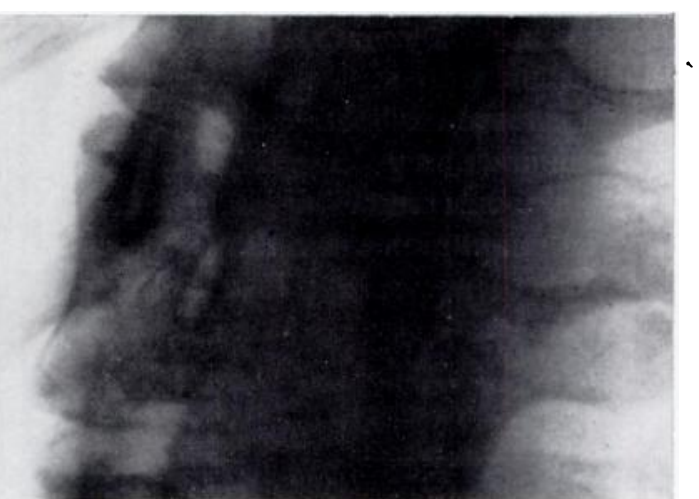

FIG. 10

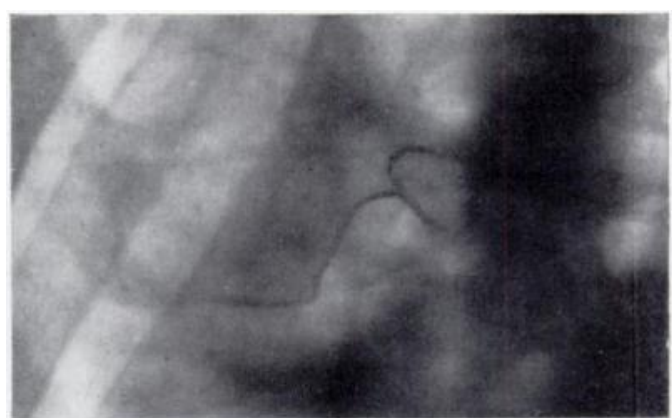

FIG. 12

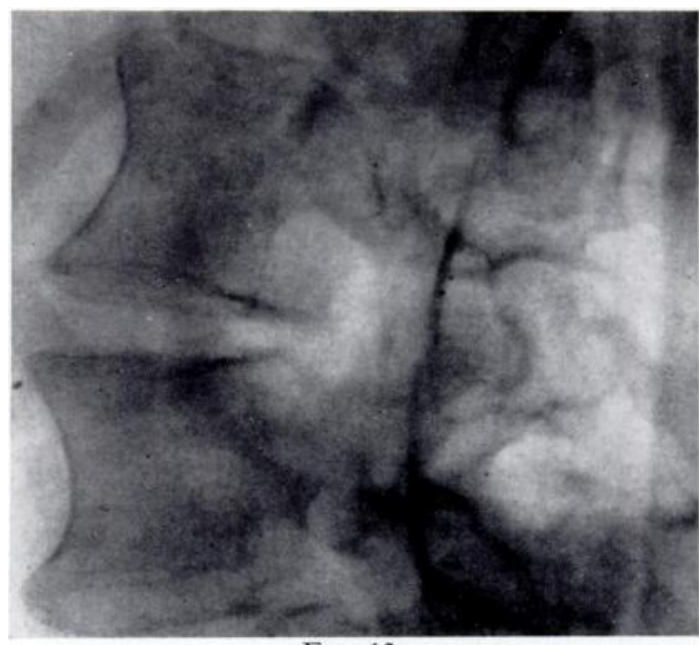

Fig. 13

The appearance of apical vertebrae in scoliosis at different rotations showing their resemblance, in Figures 9 to 13 , to radiographs of normal vertebrae at 15,30 ,

45,60 and 75 degrees of rotation respectively. Compare with Figures 2 to 6.

14 and 15). It was interesting, also, to note that neither the lordosis which is a feature of idiopathic scoliosis (Somerville 1952), nor the slight kyphosis which accompanies paralytic scoliosis, altered the radiographic appearance to any appreciable extent.

vol. 55 B, No. 3, AUGUST 1973 


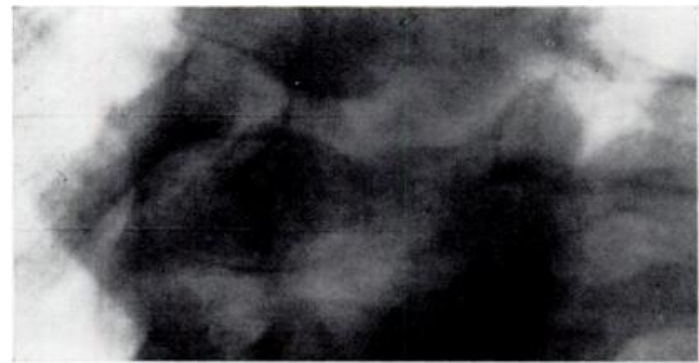

FIG. 14

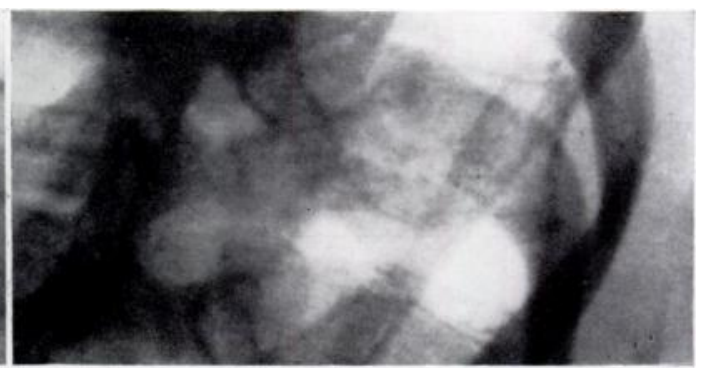

FIG. 15

Radiographs of apical vertebrae in young children with scoliosis. The radiograph in Figure 14 shows a rotation of 30 degrees in a child aged 5 years. Note the non-ossified neuro-central junction. The radiograph in Figure 15 shows a 60 -degree rotation in a 6 -year-old girl.

Although Figures 9 to 15 demonstrate that the apical vertebra at different stages of rotation projects an image which corresponds in essentials with the phases of rotation in the normal spine, it cannot be proved, nor is it claimed, that any given matching image in scoliosis represents an exactly equivalent degree of rotation as in the normal. Nonetheless, in view of their close resemblance, it is reasonable to suggest that a particular appearance of an apical vertebra represents a degree of rotation which is approximately equal to that which produces a like appearance in the normal spine.

\section{DISCUSSION}

The results have shown that it is possible to recognise each 15-degree phase of vertebral rotation in a normal spine by its characteristic radiographic appearance. They have also demonstrated the feasibility of matching the radiographic appearance of apical vertebrae in scoliosis against a series of control radiographs of normal thoracic and lumbar vertebrae to obtain a reasonable estimate of rotation in scoliosis. That the degree of rotation so obtained can only be approximate is obvious.

The value of the image-matching method lies in its ability to detect progressive vertebral rotation through 90 degrees of which none of the existing methods for estimating rotation is capable. The best known methods are those of Cobb (1948) and of Nash and Moe (1969). In Cobb's method rotation is graded by the deviation of the spinous process from the midline to the concave side of the vertebral body into five grades, ranging from 0 to $4+$; no attempt is made to correlate the grades with degrees of rotation. Nash and Moe used the convex side pedicle in a like manner, grading its progressive displacement from the convex to the concave side of the vertebral body into five grades, 0 to $4+$. They further expressed these grades as percentages of displacement ( 0 to 100 per cent) and have found a rough approximation between the percentage of pedicle displacement and the degree of vertebral rotation (Fig. 16).

Neither of these methods is entirely satisfactory for two reasons. First, although both methods use the relative position of their respective landmarks to the underlying vertebra to evaluate rotation, neither takes into account the fact that with increasing rotation from 0 to 90 degrees the outline of the vertebral body itself does not remain constant but changes from antero-posterior to lateral projection, with consequent diminution in the transverse width of the vertebral body through the last 30 degrees of rotation (Fig. 8). To describe the amount of pedicular displacement as a percentage of displacement, as suggested by Nash and Moe, without reference to the changing diameter of the vertebral body is therefore inaccurate. Secondly, Figures 1 to 8 have shown that from 0 to 30 degrees both spinous process and convex side pedicle are easily recognisable but thereafter both undergo marked alteration in appearance; the spinous process is almost impossible to identify at 45, 60 and 75 degrees and the convex side pedicle becomes a crescentic line at $\mathbf{4 5}$ degrees, is barely recognisable at 
60 degrees and is seen in lateral projection at 75 degrees. Thus both methods can be used with accuracy only up to 30 degrees of rotation but not beyond.

By marking both pedicle and transverse process of the convex side the present study has shown that the outline of the transverse process seen end-on at 45 and 60 degrees rotation is roughly similar to that of the pedicle for which, at first glance, it could be mistaken. Closer comparison of the two reveals that the transverse process outline at $\mathbf{4 5}$ degrees is circular and

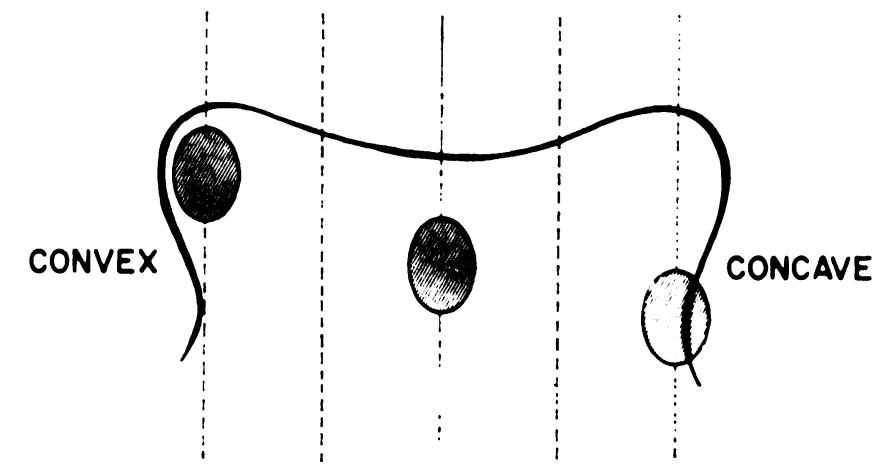

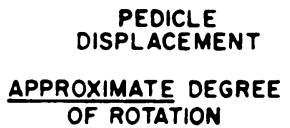

OF ROTATION

Nash and Moe's teshnique of describing vertebral rotation and estimating the degrees of rotation present. (Reprinted by permission from the Editor, Journal of Bone and Joint Surgery (1969): 51-A, 228.)

larger than the ovoid pedicle; at 60 degrees it is still larger and bean-shaped. It is submitted that what Nash and Moe describe in their method as the convex side pedicle at 50 degrees rotation and onwards is not the pedicle but the transverse process seen end-on.

The present method has overcome the disadvantages of the single landmark methods by identifying the changing dominant landmark at different stages of rotation, namely, the convex side pedicle at 15 and 30 degrees, the convex side transverse process at 45 and 60 degrees and the vertebral body at 75 and 90 degrees.

The method devised by Cherfas, Kireev and Kapustin (1965) evaluates vertebral torsion accurately but only in the initial stages of scoliosis. It has the further disadvantage of using a two-radiograph procedure which precludes its routine use in scoliosis evaluation.

There are obvious drawbacks in applying the image-matching technique to evaluate rotation in scoliosis. That the degree of estimated rotation is only an approximate and not an absolute figure has already been stated. A further disadvantage is the size of the interval between successive control radiographs: 15 degrees does represent in clinical terms quite significant deterioration in rotation before it is radiographically recognisable by this method. However, the 15-degree interval compares favourably with the 25-degree interval of Nash and Moe's method. Moreover, when serial radiographs of individual patients with scoliosis are examined, it is possible to detect intermediate stages of rotation within the 15-degree intervals described.

The effect of vertebral rotation upon the rib cage and clinical deformity is the subject of a separate study. The conviction that rotation is the most deforming element in scoliosis prompts one to submit the image-matching technique, despite its limitations, for consideration as a simple method for the routine recording of vertebral rotation at the time of measurement of the curve angle.

VOl. 55 B, NO. 3, AUGUST 1973 


\section{SUMMARY}

1. The radiographic appearance of normal thoracic and lumbar vertebrae at 15-degree intervals of rotation is described. Each image can be identified by its characteristic features. 2. The appearance on antero-posterior radiographs of apical vertebrae in scoliosis closely resembles the normal at each phase of rotation, on the basis of which an image-matching method is proposed for estimating rotation in scoliosis.

3. The method gives only approximate values of rotation but has the advantage of being able to monitor rotation through 90 degrees.

I wish to thank the Joint Finance Committee of the Institute of Orthopaedics and the Royal National Orthopaedic Hospital for a scoliosis research grant. I am grateful to Mr W. Stripp, Royal National Orthopaedic Hospital for the radiographs, and to $\mathrm{Mr} \mathbf{R}$. J. Whitley for the illustrations.

Charts depicting rotation at 15-degree intervals may be obtained on request from: The Institute of Orthopaedics, 234 Great Portland Street, London W1N 6AD.

\section{REFERENCES}

Cherfas, M. D., Kireev, V. A., and Kapustin, A. S. (1965): Metodika opredeleniia torsii pozvonkov pri nachal'nykh formakh skolioza. Ortopediya, Travmatologiya i Protezirovanie, 26, 30.

Совв, J. R. (1948): Outline for the Study of Scoliosis. American Academy of Orthopaedic Surgeons, Instructional Course Lectures, 5, 261.

Ferguson, A. B. (1949): Roentgen Diagnosis of the Extremities and Spine. 2nd Edition. New York: Paul B. Hoeber, Inc.

NASH, C. L., Jun., and MoE, J. H. (1969): A Study of Vertebral Rotation. Journal of Bone and Joint Surgery, 51-A, 223.

SOMERville, E. W. (1952): Rotational Lordosis: The Development of the Single Curve. Journal of Bone and Joint Surgery, 34-B, 421. 